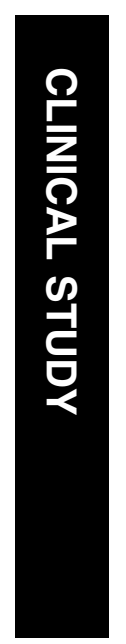

\title{
Comparison of laser ray-tracing and skiascopic ocular wavefront-sensing devices
}

'Department of Ophthalmology, University of California San Diego, Shiley Eye Center, La Jolla, CA, USA

${ }^{2}$ Department of Visual Science, Osaka University Graduate School of Medicine, Suita, Osaka, Japan

${ }^{3}$ Codet Eye Clinic, Tijuana, Mexico

Correspondence:

D-UG Bartsch,

Department of

Ophthalmology, University

of California San Diego,

Shiley Eye Center,

9415 Campus Point Drive,

La Jolla,

CA 92093-0946, USA

Tel: + 18585343842 ;

Fax: + 18585347985 .

E-mail: dbartsch@

ucsd.edu

Received: 1 March 2007

Accepted in revised form: 15 May 2007

Published online: 15 June 2007

This study was presented in the annual meeting of Association of Research in Vision and Ophthalmology, 4-9 May 2003, Fort Lauderdale, FL, USA

Conflict of interest These authors state no conflict of interest

\begin{abstract}
Purpose To compare two wavefront-sensing devices based on different principles. Methods Thirty-eight healthy eyes of 19 patients were measured five times in the reproducibility study. Twenty eyes of 10 patients were measured in the comparison study. The Tracey Visual Function Analyzer (VFA), based on the ray-tracing principle and the Nidek optical pathway difference (OPD)-Scan, based on the dynamic skiascopy principle were compared. Standard deviation (SD) of root mean square (RMS) errors was compared to verify the reproducibility. We evaluated RMS errors, Zernike terms and conventional refractive indexes ( $\mathrm{Sph}, \mathrm{Cyl}, \mathrm{Ax}$, and spherical equivalent).

Results In RMS errors reading, both devices showed similar ratios of SD to the mean measurement value (VFA: $\mathbf{5 7 . 5} \pm \mathbf{1 1 . 7 \%}$, OPD-Scan: $53.9 \pm 10.9 \%)$. Comparison on the same eye showed that almost all terms were significantly greater using the VFA than using the OPD-Scan. However, certain high spatial frequency aberrations (tetrafoil, pentafoil, and hexafoil) were consistently measured near zero with the OPD-Scan.

Conclusion Both devices showed similar level of reproducibility; however, there was considerable difference in the wavefront reading between machines when measuring the same eye. Differences in the number of sample points, centration, and measurement algorithms between the two instruments may explain our results.

Eye (2008) 22, 1384-1390; doi:10.1038/sj.eye.6702901; published online 15 June 2007
\end{abstract}

Keywords: skiascopy; ray tracing; higher order aberrations; wavefront sensing
D-UG Bartsch1, K Bessho ${ }^{1,2}$, L Gomez ${ }^{3}$ and WR Freeman ${ }^{1}$
Introduction

Retinal imaging is an important objective method to assess retinal structures. The many optical aberrations in the human eye limit the resolution of retinal photographic equipment. Thus, the transverse resolution in retinal photography is of the order of $15-20 \mu \mathrm{m}$ while the axial longitudinal resolution is of the order of 300-450 $\mu \mathrm{m} .{ }^{1}$ Development of high-resolution fundus imaging will improve our understanding and possibly treatment of many retinal disorders.

Adaptive optics in astronomy dynamically compensates for the wavefront error caused by atmospheric turbulences, ${ }^{2,3}$ and, recently, it has been applied to compensate for aberrations of the human eye $e^{4,5}$ and may be used to obtain higher resolution retinal images. ${ }^{6-9}$

An adaptive optical instrument requires a device for measuring aberrations. Since neither a wavefront sensor (WS) nor a point light source can be placed inside the eye, a single pass set-up as is used in optical engineering is not feasible in the living human eye. To circumvent this limitation one may use a bright light source to project a relatively small point onto the retinal surface. ${ }^{4}$ A WS, such as a Hartmann-Shack sensor then captures the light and calculates the aberrations, assuming the reflected light is similar to the light originating from a point source. Alternatively, a series or a bundle of equidistant rays may be used to create an image of the wavefront on the retinal surface. ${ }^{10}$ The image of the ray pattern is captured and used to recreate a wavefront measurement. A third method is skiascopy in which a light slit scans across the pupil, similar to retinoscopy, while a series of photodiode records the change in light intensity over slit location, and the aberrations are determined mathematically. ${ }^{11,12}$ 
Two commercially available examples of such wavefront instruments are the ray-tracing method incorporated in the Tracey Visual Function Analyzer (VFA, Tracey Technologies, Houston, TX, USA) and the skiascopy method used in the Nidek optical pathway difference (OPD)-Scan (OPD-Scan Refractive Power/ Corneal Analyzer ARK-10000, Nidek, Gamagori, Japan). In this study, we determined the reproducibility of both methods to measure the wavefront in normal human eyes. Additionally, we compared the wavefront measurements in normal human eyes.

\section{Materials and methods}

\section{Subjects}

To verify the reproducibility of devices in the first study, 20 normal eyes of 10 patients (4 men, 6 women; age $32.9 \pm 7.1$ years; mean $\pm \mathrm{SD}$ ) were examined using the VFA. The Nidek OPD-Scan arm of the study consisted of another cohort of 18 eyes of 9 normal patients ( 2 men, 7 women; age $32.6 \pm 7.1$ ) In a second study, the two instruments were compared directly by measuring another 20 healthy eyes (4 men, 6 women; age $32.8 \pm 6.8$ ) sequentially within $30 \mathrm{~min}$ of each other and in random instrument order.

\section{Instruments}

The OPD-Scan is based on the dynamic skiascopy principle. ${ }^{11,12}$ An infrared light slit and photodetectors are placed on a rotating wheel along the same rotational position across the pupil. By rotating the wheel, the instrument measures the time for light to peak at each photodiode, and by comparing the results with the theoretical reference time it calculates the OPD, and subsequently wavefront error. This procedure yields 1440 data points within $0.4 \mathrm{~s}$. In the default setting, the device scans the eye at a $6 \mathrm{~mm}$ scan diameter, and displays wavefront error at both 5 and $3 \mathrm{~mm}$ concentric area as well as conventional refractive indexes. The OPD-Scan is a hybrid of autorefractor and wavefront device working independently; the device provides two conventional refractive indexes independently measured. A complete eye examination consists of three separate OPD maps and one corneal topography, which requires about 1-2 min per eye.

The Tracey VFA (software version 1.0) is based on the ray-tracing principle. The device projects 64 light rays in programmable scan pattern (in default on four concentric circles) across the pupil in succession and detects the retinal location of reflection with the help of two linear charged coupled device (CCD) arrays. ${ }^{13,14}$ On the basis of these measurements, the device calculates the retinal point spread function and subsequently the wavefront aberrations. In default setting of automatic mode, it scans at the largest possible pupil area and displays wavefront data. Conventional refractive indexes are generated by reverse calculation of Zernike coefficients, and therefore values depend on the size of analysis area. A single measurement constitutes a complete exam for each eye, which takes $0.06 \mathrm{~s}$ per eye.

Both devices were maintained under manufacture's proper calibration and after completion of the study, calibration of both devices was verified and confirmed to working correctly.

\section{Measurements}

\section{Reproducibility study}

The VFA measurements were done at maximum non-pharmacologically dilated pupil size in a dimly lit room and the actual scan size ranged between 5.5 and $6.1 \mathrm{~mm}(5.8 \pm 0.2 \mathrm{~mm})$. This device requires the operator to project the 64-point pattern through the largest pupil size available. For the OPD-Scan arm of the study, the same illumination and patient pupil size was used, however, the OPD-Scans analysed only the central 6-mm diameter of the pupil. In both studies, arms' measurements were performed in a dark room, without dilation. Each eye was scanned five times, and Zernike coefficients were obtained up to the sixth order.

\section{Direct comparison study}

After completion of the reproducibility study, we decided to conduct a direct comparison between both instruments. Each eye was examined once with two devices in succession without dilation, and the order of device was randomized. The measurement area was fixed at $4 \mathrm{~mm}$ diameter for both devices. Three refractive data sets from the VFA, autorefracto-keratometer (ARK), and WS integrated in the OPD-Scan were compared as well as the Zernike coefficients.

Since refractive indexes obtained using the VFA depend on analysis area size, a 3-mm analysis area was used to calculate indexes. Refractive indexes given by the ARK and the OPD-Scan were obtained from the same analysis area, $3 \mathrm{~mm}$.

\section{Analysis}

All data from both instruments were analysed using JMP IN ver. 4.0.2 (SAS Institute Inc., Cary, NC, USA).

We used an equation described by Schwiegerling ${ }^{15}$ for the use of coefficients up to sixth order to compare the data scanned at different pupil sizes into a unified pupil size. ${ }^{16,17}$ The reason for using this pupil size transformation is to be able to do a direct comparison 
between the measurements for both instruments. This method is an acceptable method of comparing results from two devices with different scan diameter. This method cannot predict the wavefront of larger analysis areas; the new analysis area is forced to be smaller than the original area.

In the first study (reproducibility of devices), there were small deviations of the scanning area in the VFA arm because of the automatic acquisition mode.

Therefore, all obtained data in both arms were transformed using the above-mentioned equation into a unified analysis area size of $5 \mathrm{~mm}$ diameter. Root mean square (RMS) errors were calculated from transformed coefficients and the standard deviation (SD) of each five RMS errors per eye was calculated.

In the direct comparison study, the VFA was used in manual acquisition mode and the scanning area of both devices was kept uniform at $4 \mathrm{~mm}$ diameter; thus, the RMS errors were calculated from the raw data.

In both studies, the analysis was performed by eye and also using one eye per individual (by using right eye only and left eye only). In each examination, we were careful to clinically align the eyes for optical centration and torsional alignment.

The study was approved by the UCSD Human Research Protection Program and informed consent was obtained. We certify that all applicable institutional and governmental regulations concerning the ethical use of human volunteers were followed during this research.

\section{Results}

\section{Reproducibility study}

Equivalent spherical refractive error ranged between -0.8 and $0.9 \mathrm{D}(-0.1 \pm 0.8 \mathrm{D})$ for the VFA arm and -1.3 and $0.0 \mathrm{D}(-0.5 \pm 0.5 \mathrm{D})$ for the OPD-Scan arm. In the analysis of the RMS errors, we found that the SD of the OPD-Scan was significantly lower for all 25 indexes (Table 1). Interestingly, when we calculated the ratio of the SD to the mean RMS value, we found that there was almost no difference (VFA: $57.5 \pm 11.7 \%$, OPD-Scan: $53.9 \pm 10.9 \%$ of mean value; $(P=0.153, t$-test $)$; Figure 1$)$. Similar tendencies were found for several combined indexes describing coma-like, spherical-like, and total higher order aberration. ${ }^{18-20}$

The only exception was in the measurements with the OPD-Scan at certain higher order Zernike terms where the absolute values measured by the OPD-Scan were at almost zero value $(<0.0001)$. These terms were several high spatial frequency indexes, namely C10 $\left(C_{4}^{-4}\right), \mathrm{C} 14$ $\left(C_{4}^{4}\right), \mathrm{C} 15\left(C_{5}^{-5}\right), \mathrm{C} 20\left(C_{5}^{5}\right), \mathrm{C} 21\left(C_{6}^{-6}\right), \mathrm{C} 22\left(C_{6}^{-4}\right), \mathrm{C} 26\left(C_{6}^{4}\right)$, and $\mathrm{C} 27\left(C_{6}^{6}\right)$. These terms are typically described as tetrafoil, pentafoil, and hexafoil. Similar tendencies were found when the analysis was performed using one eye per individual; ie either all right or all left eyes (data not shown).

Additionally, we also performed the same analyses without the equation transforming data to unified pupil size. Using uncorrected data at various pupil sizes $(5.8 \pm 0.2 \mathrm{~mm})$, the results were similar (data not shown).

\section{Direct comparison study}

\section{Conventional refraction}

There was no significant difference between the conventional refraction measured by ARK and WS integrated in the OPD-Scan, although these devices indicated different absolute values (Table 2).

The readings of the Tracey device (VFA) were significantly more hyperopic and more astigmatic than by either ARK or WS in the OPD-Scan. Similar tendencies were found when the analysis was performed using one eye per individual (data not shown).

\section{Wavefront measurement}

The RMS errors between Z3 and Z27 (except Z4), as well as the combined indexes, were significantly greater from the VFA than the OPD-Scan (Table 3). Similar to what we found in the reproducibility study, readings of several high spatial frequency terms using the OPD-Scan showed extremely low absolute values $(<0.0001)$. Similar tendencies were found when the analysis was performed using one eye per individual (data not shown).

\section{Discussion}

We compared two methods of double-pass wavefrontmeasuring devices to determine their reproducibility. Reproducibility is not synonymous with accuracy and assessment of intra- and inter-machine variability does not determine which device, if either, is accurately measuring the wavefront. We determined intra-machine variability in two groups of normal subjects. For the direct comparison between the two instruments, we used the same group of subjects. We limited our statistical analysis to the evaluation of the Zernike coefficients as we are mainly interested in higher order aberrations. We performed our analysis by eye as the study was performed to evaluate machine measurements and each eye was an independent entity in this study.

Serial ray-tracing technology is incorporated into the Tracey VFA instrument, where one point is measured at a time. Photodetectors detect the location of where each light ray strikes the retina. This may produce less error in eyes with large aberrations, compared to the Hartmann-Shack device. The limitation of the VFA is 
Table 1 Standard deviation of five measurements and its ratio to mean measurement value

\begin{tabular}{|c|c|c|c|c|c|c|c|}
\hline \multirow[t]{2}{*}{ Analysis area: $5 \mathrm{~mm}$} & \multicolumn{3}{|c|}{$V F A$} & \multicolumn{3}{|c|}{ OPD-Scan } & \multirow[b]{2}{*}{ P-value } \\
\hline & Mean of $S D$ & $\begin{array}{c}\text { Mean of } \\
\text { mean }\end{array}$ & $\begin{array}{c}\text { Ratio SD:mean } \\
(\%)\end{array}$ & Mean of SD & $\begin{array}{c}\text { Mean of } \\
\text { mean }\end{array}$ & $\begin{array}{c}\text { Ratio SD:mean } \\
(\%)\end{array}$ & \\
\hline Sph (D) & 0.2 & 0.4 & $\mathrm{~N} / \mathrm{A}$ & 0.2 & -0.3 & $\mathrm{~N} / \mathrm{A}$ & $0.573^{\mathrm{b}}$ \\
\hline Cyl (D) & 0.1 & -0.8 & $\mathrm{~N} / \mathrm{A}$ & 0.1 & -0.4 & $\mathrm{~N} / \mathrm{A}$ & $0.796^{\mathrm{b}}$ \\
\hline Ax (deg) & 7.1 & 102.7 & $\mathrm{~N} / \mathrm{A}$ & 8.8 & 128.1 & $\mathrm{~N} / \mathrm{A}$ & $0.110^{\mathrm{b}}$ \\
\hline Spherical equivalent (D) & 0.2 & -0.1 & $\mathrm{~N} / \mathrm{A}$ & 0.1 & -0.5 & $\mathrm{~N} / \mathrm{A}$ & $0.749^{\mathrm{b}}$ \\
\hline $\mathrm{Z} 3$ & 0.054 & 0.240 & 43.0 & 0.019 & 0.075 & 45.4 & 0.942 \\
\hline $\mathrm{Z} 4$ & 0.121 & 0.426 & 38.6 & 0.086 & 0.326 & 37.3 & 0.737 \\
\hline Z5 & 0.069 & 0.355 & 26.0 & 0.024 & 0.088 & 37.6 & 0.204 \\
\hline Z6 & 0.033 & 0.107 & 46.7 & 0.017 & 0.037 & 58.1 & 0.342 \\
\hline $\mathrm{Z7}$ & 0.038 & 0.095 & 49.3 & 0.007 & 0.024 & 48.6 & 0.272 \\
\hline Z8 & 0.031 & 0.054 & 62.0 & 0.007 & 0.019 & 46.2 & 0.042 \\
\hline Z9 & 0.029 & 0.064 & 48.1 & 0.014 & 0.033 & 53.4 & 0.421 \\
\hline Z10 & 0.014 & 0.026 & 62.4 & $-^{c}$ & $-^{c}$ & $\mathrm{~N} / \mathrm{A}$ & $\mathrm{N} / \mathrm{A}$ \\
\hline Z11 & 0.016 & 0.024 & 67.0 & 0.003 & 0.006 & 56.3 & 0.299 \\
\hline Z12 & 0.021 & 0.062 & 46.8 & 0.007 & 0.023 & 39.5 & 0.421 \\
\hline Z13 & 0.019 & 0.034 & 56.3 & 0.004 & 0.009 & 58.4 & 0.804 \\
\hline Z14 & 0.017 & 0.040 & 52.0 & $-^{c}$ & $-^{c}$ & $\mathrm{~N} / \mathrm{A}$ & $\mathrm{N} / \mathrm{A}$ \\
\hline Z15 & 0.006 & 0.009 & 70.0 & $-^{c}$ & $-^{c}$ & $\mathrm{~N} / \mathrm{A}$ & $\mathrm{N} / \mathrm{A}$ \\
\hline Z16 & 0.005 & 0.008 & 60.5 & 0.002 & 0.003 & 72.3 & 0.342 \\
\hline Z17 & 0.008 & 0.013 & 68.5 & 0.002 & 0.004 & 52.1 & 0.045 \\
\hline Z18 & 0.005 & 0.009 & 65.6 & 0.002 & 0.003 & 56.3 & 0.313 \\
\hline Z19 & 0.004 & 0.007 & 63.9 & 0.002 & 0.003 & 67.6 & 0.569 \\
\hline $\mathrm{Z} 20$ & 0.004 & 0.009 & 52.4 & $-^{c}$ & $-^{c}$ & $\mathrm{~N} / \mathrm{A}$ & $\mathrm{N} / \mathrm{A}$ \\
\hline $\mathrm{Z} 21$ & 0.003 & 0.004 & 77.5 & $-^{c}$ & $-^{c}$ & $\mathrm{~N} / \mathrm{A}$ & $\mathrm{N} / \mathrm{A}$ \\
\hline $\mathrm{Z} 22$ & 0.003 & 0.004 & 65.6 & $-^{c}$ & $-^{c}$ & $\mathrm{~N} / \mathrm{A}$ & $\mathrm{N} / \mathrm{A}$ \\
\hline $\mathrm{Z} 23$ & 0.004 & 0.005 & 72.4 & 0.001 & 0.001 & 72.5 & 0.781 \\
\hline Z24 & 0.005 & 0.009 & 58.2 & 0.002 & 0.004 & 62.8 & 0.492 \\
\hline $\mathrm{Z} 25$ & 0.004 & 0.007 & 59.0 & 0.001 & 0.001 & 51.1 & 0.492 \\
\hline Z26 & 0.003 & 0.005 & 66.2 & $-^{c}$ & - $^{c}$ & $\mathrm{~N} / \mathrm{A}$ & $\mathrm{N} / \mathrm{A}$ \\
\hline Z27 & 0.004 & 0.007 & 60.5 & $-^{c}$ & $-^{c}$ & $\mathrm{~N} / \mathrm{A}$ & $\mathrm{N} / \mathrm{A}$ \\
\hline S2 (RMS of C3-C5) & 0.104 & 0.686 & 16.7 & 0.083 & 0.370 & 23.2 & 0.456 \\
\hline S3 (RMS of C6-C9) & 0.039 & 0.202 & 20.1 & 0.016 & 0.068 & 26.8 & 0.273 \\
\hline S4 (RMS of C10-C14) & 0.022 & 0.103 & 20.8 & 0.007 & 0.028 & 28.2 & 0.286 \\
\hline S5 (RMS of C15-C20) & 0.009 & 0.027 & 29.0 & 0.002 & 0.008 & 30.2 & 0.609 \\
\hline S6 (RMS of C21-C27) & 0.006 & 0.019 & 23.3 & 0.001 & 0.004 & 37.5 & 0.093 \\
\hline S3 + S5 (RMS of S3 and S5) & 0.039 & 0.205 & 19.7 & 0.016 & 0.069 & 26.6 & 0.184 \\
\hline S4 + S6 (RMS of S4 and S6) & 0.022 & 0.105 & 20.3 & 0.007 & 0.028 & 27.0 & 0.388 \\
\hline Total HO (RMS of S3-S6) & 0.040 & 0.238 & 16.2 & 0.016 & 0.076 & 23.0 & 0.150 \\
\hline Total RMS (RMS of S2-S6) & 0.105 & 0.746 & 15.3 & 0.082 & 0.384 & 20.3 & 0.373 \\
\hline
\end{tabular}

RMS, root mean square; SD, standard deviation.

Unit: millimetre unless specified.

Mean of mean: average of each eye's averaged measured value on five exams.

Mean of SD: average of each eye's SD on five exams.

Ratio SD:mean: average of each eye's (mean of SD/mean of mean).

Z3 to Z27: RMS of each Zernike coefficients (C3-C27)

S2: second order, defocus and astigmatism; S3: third order, coma and trefoil; S4: fourth order, tetrafoil and spherical; S5: fifth order, coma, tetrafoil, and pentafoil; S6: sixth order, spherical, tetrafoil, and hexafoil; S3 + S5: coma-like aberration; S4 + S6: spherical-like aberration.

${ }^{a}$ Wilcoxon rank-sum test on ratio SD:mean.

${ }^{b}$ Wilcoxon rank-sum test on SD.

'Value was below 0.0001 .

that the point-spread function on the retina is sampled by two linear CCD arrays, but not by a true two-dimensional detector, while the advantage appears to be the high acquisition speed at reduced equipment cost. The device we tested measures aberrations over $60 \mathrm{~ms}$ which is extremely rapid and which may cause less susceptibility to eye motion and tear film artefacts. Since this study was performed, newer devices from this company have become available which capture 95 points and over 200 points. Such instrument may improve the spatial resolution of the device. Liang and co-workers ${ }^{21}$ have published a comparison of the three wavefront devices based on the Hartmann-Shack WS. They studied the WaveScan (Visx Inc., Santa Clara, CA, USA), the 


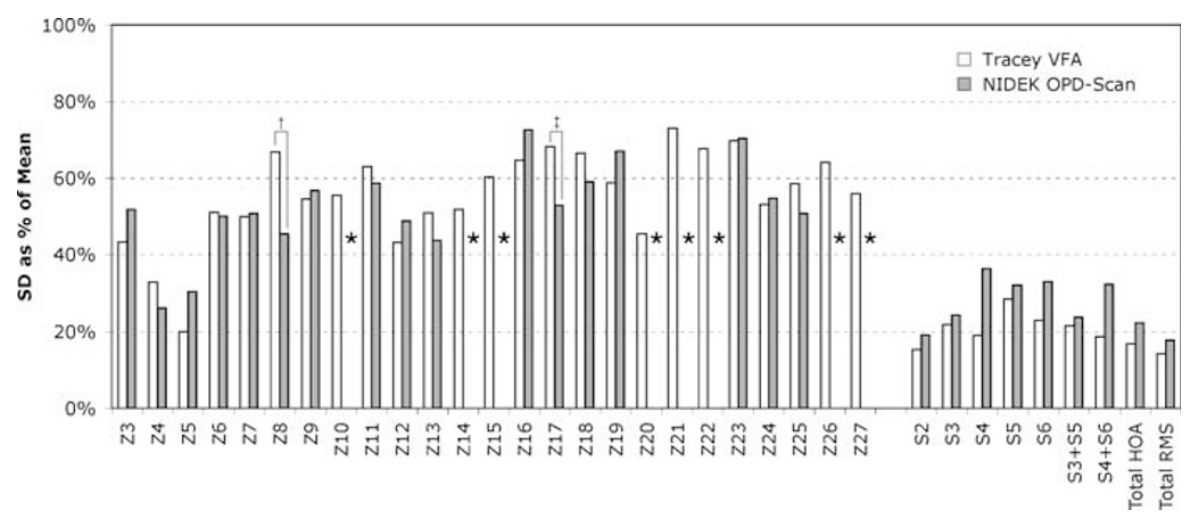

Figure 1 SD as percent of mean measurement value. SD was within $80 \%$ of mean value in both devices and only Z8 (RMS of $C_{3}^{1}$ ) and Z17 $\left(C_{5}^{-1}\right)$ showed significant difference of two devices $(P=0.042 \dagger, 0.045 \ddagger)$. ${ }^{*}$ Both SD and mean value were below 0.0001 and ratio was not calculated.

Table 2 Conventional refraction measured by each device (direct comparison study)

\begin{tabular}{lccrr}
\hline Mean $\pm S D$ & Sph $(D)$ & $C y l(D)$ & Ax (deg) & Spherical equivalent $(D)$ \\
\hline VFA & $0.7 \pm 0.5$ & $-1.1 \pm 0.8$ & $97.9 \pm 75.8$ & $0.1 \pm 0.6$ \\
OPD-Scan & $-0.1 \pm 0.4$ & $-0.8 \pm 0.8$ & $100.7 \pm 66.9$ & $-0.5 \pm 0.6$ \\
ARK & $-0.1 \pm 0.4$ & $-0.7 \pm 0.9$ & $103.4 \pm 66.0$ & $-0.5 \pm 0.6$ \\
& & & & $<.295$ \\
VFA vs OPD-Scan* & $<\mathbf{0 . 0 0 1}$ & 0.051 & 0.586 & $<\mathbf{0 0 1}$ \\
ARK vs OPD-Scan* & 0.222 & 0.136 & 0.188 & 0.634 \\
VFA vs ARK* & $<\mathbf{0 . 0 0 1}$ & $\mathbf{0 . 0 0 1}$ & $<\mathbf{0 . 0 0 1}$ \\
\hline
\end{tabular}

ARK, autorefractometer (OPD-Scan built-in device, working independently); OPD-Scan, optical pathway difference Scan; SD, standard deviation; VFA, Visual Function Analyzer.

${ }^{*} P$-values of paired $t$-test on measured values. Bold values indicate significant difference.

LADARWave (Alcon Inc., Fort Worth, TX, USA), and the Zywave (Bausch \& Lomb Inc., Rochester, NY, USA). These investigators found significant discrepancies between these three devices in higher order aberrations.

The skiascopy instrument (Nidek Inc., Fremont, CA, USA, OPD) has an apparent limitation that skewed rays may not be detected. Returned light rays that intersect the exit pupil orthogonal to the photodetector array rotation may not properly be detected. This may explain the inability to measure the higher spatial frequency components of the wavefront (tetrafoil, pentafoil, and hexafoil) in our study.

The OPD-Scan requires recording of three complete OPD maps and one corneal topography which takes several minutes; much longer than the VFA. The technique is more akin to automated retinoscopy at multiple time points and, although not a true wavefront measurement, it generates a refractive error map that can be translated to a wavefront measurement.

Both instruments gave equally reproducible results. There have been several other studies of wavefront measurement device reproducibility. ${ }^{13,14,22}$ These have included the Tracey device and the WaveScan system (VISX, Santa Clara, CA, USA) as well as a method using
Hartmann-Shack techniques. These studies found that the standard deviation of the conventional refractive index measured by wavefront devices was between 0.1 and $0.5 \mathrm{D}$. We also found that both machines produced similar levels of SD (Table 1). However, since these other studies mainly focused on the conventional refraction, our goal was to study higher order aberrations. We found that the SD of the OPD-Scan was generally lower than the VFA, however the actual measured values were also smaller; the ratio of SD to the measured value was thus similar between the two machines for most of the indexes.

As an indicator of reproducibility, the SD of several repeat measurements as well as multiplication of SD by a certain constant depending on the sample size has been considered. The difference between an entire session measuring the same population in different timeline has also been considered. ${ }^{23-26}$ Gobbe and co-workers ${ }^{23}$ also suggested relative repeatability, which is a ratio of SD to the measured values in their study on corneal wavefront aberration. When we take the raw SD values as the magnitude of reproducibility, our data suggest that the OPD-Scan might be more reproducible than the Tracey device. However, the ratio of SD to the mean measured 
Table 3 RMS errors obtained using both devices (direct comparison study)

\begin{tabular}{|c|c|c|c|c|}
\hline & $\begin{array}{c}V F A \\
M e a n \pm S D\end{array}$ & $\begin{array}{l}O P D-S c a n \\
M e a n \pm S D\end{array}$ & $\begin{array}{c}\% \text { Difference } \\
(V F A-O P D-S c a n) / V F A\end{array}$ & $\mathrm{P}-$ value ${ }^{\mathrm{a}}$ \\
\hline $\mathrm{Z3}$ & $0.140 \pm 0.100$ & $0.048 \pm 0.039$ & $61.0 \pm 27.5$ & $<0.001$ \\
\hline $\mathrm{Z} 4$ & $0.269 \pm 0.219$ & $0.182 \pm 0.157$ & $-41.8 \pm 201.5$ & 0.093 \\
\hline Z5 & $0.420 \pm 0.314$ & $0.107 \pm 0.131$ & $71.2 \pm 22.7$ & $<0.001$ \\
\hline Z6 & $0.049 \pm 0.036$ & $0.028 \pm 0.033$ & $26.2 \pm 105.2$ & 0.007 \\
\hline $\mathrm{Z7}$ & $0.040 \pm 0.029$ & $0.012 \pm 0.007$ & $35.7 \pm 82.8$ & 0.001 \\
\hline Z8 & $0.039 \pm 0.028$ & $0.014 \pm 0.013$ & $-466.0 \pm 1700.6$ & 0.003 \\
\hline Z9 & $0.044 \pm 0.041$ & $0.020 \pm 0.016$ & $-32.9 \pm 170.0$ & 0.025 \\
\hline $\mathrm{Z} 10$ & $0.021 \pm 0.015$ & $-\mathrm{b}$ & $\mathrm{N} / \mathrm{A}$ & $\mathrm{N} / \mathrm{A}$ \\
\hline Z11 & $0.015 \pm 0.013$ & $0.005 \pm 0.004$ & $39.7 \pm 59.6$ & 0.001 \\
\hline $\mathrm{Z} 12$ & $0.034 \pm 0.023$ & $0.011 \pm 0.007$ & $13.0 \pm 190.9$ & 0.002 \\
\hline $\mathrm{Z} 13$ & $0.020 \pm 0.018$ & $0.009 \pm 0.010$ & $-221.1 \pm 849.7$ & 0.040 \\
\hline Z14 & $0.022 \pm 0.014$ & $-\mathrm{b}$ & $\mathrm{N} / \mathrm{A}$ & $\mathrm{N} / \mathrm{A}$ \\
\hline Z15 & $0.006 \pm 0.004$ & $-^{\mathrm{b}}$ & $\mathrm{N} / \mathrm{A}$ & $\mathrm{N} / \mathrm{A}$ \\
\hline Z16 & $0.007 \pm 0.005$ & $0.002 \pm 0.001$ & $49.7 \pm 68.4$ & $<0.001$ \\
\hline Z17 & $0.006 \pm 0.005$ & $0.002 \pm 0.002$ & $52.1 \pm 60.8$ & 0.001 \\
\hline Z18 & $0.009 \pm 0.008$ & $0.003 \pm 0.003$ & $48.0 \pm 66.6$ & 0.001 \\
\hline Z19 & $0.005 \pm 0.004$ & $0.002 \pm 0.001$ & $-43.4 \pm 250.6$ & 0.004 \\
\hline $\mathrm{Z} 20$ & $0.006 \pm 0.005$ & ${ }^{b}$ & $\mathrm{~N} / \mathrm{A}$ & $\mathrm{N} / \mathrm{A}$ \\
\hline $\mathrm{Z} 21$ & $0.005 \pm 0.004$ & $-^{\mathrm{b}}$ & $\mathrm{N} / \mathrm{A}$ & $\mathrm{N} / \mathrm{A}$ \\
\hline $\mathrm{Z} 22$ & $0.005 \pm 0.003$ & $\ldots^{\mathrm{b}}$ & $\mathrm{N} / \mathrm{A}$ & $\mathrm{N} / \mathrm{A}$ \\
\hline $\mathrm{Z} 23$ & $0.004 \pm 0.003$ & $0.001 \pm 0.002$ & $60.5 \pm 37.7$ & $<0.001$ \\
\hline Z24 & $0.007 \pm 0.006$ & $0.000 \pm 0.001$ & $93.7 \pm 8.9$ & $<0.001$ \\
\hline $\mathrm{Z} 25$ & $0.006 \pm 0.004$ & $0.002 \pm 0.003$ & $21.0 \pm 101.7$ & 0.004 \\
\hline Z26 & $0.006 \pm 0.004$ & $-^{b}$ & $\mathrm{~N} / \mathrm{A}$ & $\mathrm{N} / \mathrm{A}$ \\
\hline $\mathrm{Z} 27$ & $0.004 \pm 0.003$ & $-^{b}$ & $\mathrm{~N} / \mathrm{A}$ & $\mathrm{N} / \mathrm{A}$ \\
\hline S2 & $0.566 \pm 0.318$ & $0.234 \pm 0.187$ & $53.1 \pm 30.5$ & $<0.001$ \\
\hline S3 & $0.097 \pm 0.050$ & $0.045 \pm 0.031$ & $50.1 \pm 31.2$ & $<0.001$ \\
\hline S4 & $0.060 \pm 0.024$ & $0.017 \pm 0.009$ & $60.3 \pm 41.1$ & $<0.001$ \\
\hline S5 & $0.020 \pm 0.008$ & $0.005 \pm 0.003$ & $70.0 \pm 19.4$ & $<0.001$ \\
\hline S6 & $0.017 \pm 0.006$ & $0.003 \pm 0.003$ & $78.9 \pm 20.5$ & $<0.001$ \\
\hline S3+S5 & $0.100 \pm 0.050$ & $0.046 \pm 0.031$ & $51.4 \pm 29.6$ & $<0.001$ \\
\hline $\mathrm{S} 4+\mathrm{S} 6$ & $0.062 \pm 0.023$ & $0.018 \pm 0.009$ & $64.5 \pm 29.8$ & $<0.001$ \\
\hline Total HO & $0.121 \pm 0.045$ & $0.051 \pm 0.030$ & $57.1 \pm 22.5$ & $<0.001$ \\
\hline Total RMS & $0.585 \pm 0.310$ & $0.244 \pm 0.183$ & $53.5 \pm 27.0$ & $<0.001$ \\
\hline
\end{tabular}

OPD-Scan, optical pathway difference Scan; RMS, root mean square; SD, standard deviation; VFA, Visual Function Analyzer.

a Paired Wilcoxon rank-sum test on measured values.

bBoth mean and SD were below 0.0001 .

value suggests that both devices have similar reproducibility.

Direct comparisons on the same day between the two instruments on the same eyes showed different Zernike coefficients. It is not possible to determine which device measures the true wavefront value. The differences between the two instruments were more apparent in higher spatial frequency terms. We did not compare the measurements for which the OPD-Scan values were extremely low. The fact that the ray-tracing instruments perform measurements over a much shorter interval of time than the skiascopy instrument ( $0.06 \mathrm{~s}$ vs $1-2 \mathrm{~min}$ ), and the fact that the VFA allows the analysis of a single measurement suggests that the VFA may be better at avoiding motion or tear film artefacts; however, skiascopy measures far more points (1440 vs 64) than the VFA.
If one defines accuracy as adhering to the true value and precision as arriving at a value with high reproducibility, we determined that both instruments are precise, but both cannot be accurate. Reading of conventional refraction suggests that OPD-Scan has better accuracy than VFA in comparison with the ARK; however, for higher order aberrations, we cannot determine which instrument is more accurate. The direct comparison study showed a systematic difference in the results between both instruments. Higher order aberrations have no known correlation to clinical optical practice, and thus, it has been difficult to perform absolute calibration of higher order aberrations in WSs. The results of our studies emphasize the need for absolute calibration of higher order aberrations in WSs. 


\section{Acknowledgements}

This study was sponsored in part by a grant from the Osaka Medical Research Foundation for Incurable Diseases, Osaka, Japan, (Bessho K), National Eye Institute NIH-NEI Grants EY13304 and EY16323 (Bartsch DU), NIH Grant EY07366 (Freeman WR) and Research to Prevent Blindness (UCSD).

\section{References}

1 Bartsch DU, Freeman WR. Axial distribution analysis of the human retina with a confocal scanning laser tomograph. Exp Eye Res 1994; 58: 161-173.

2 Babcock HW. The possibility of compensating astronomical seeing. Publ Astron Soc Pac 1953; 65: 229-236.

3 Tyson RK. Adaptive optics system performance approximations for atmospheric turbulence correction. Opt Eng 1990; 29(10): 1165-1173.

4 Liang J, Grimm B, Goelz S, Bille JF. Objective measurement of wave aberrations of the human eye with the use of a Hartmann-Shack wave-front sensor. J Opt Soc Am A Opt Image Sci Vis 1994; 11(7): 1949-1957.

5 Zhu LJ, Bartsch DU, Freeman WR, Sun PC, Fainman Y. Modeling human eye aberrations and their compensation for high-resolution retinal imaging. Optom Vis Sci 1998; 75(11): 827-839.

6 Hofer H, Chen L, Yoon GY, Singer B, Yamauchi Y, Williams DR. Improvement in retinal image quality with dynamic correction of the eye's aberrations. Optic Express 2001; 8(11): 631-643.

7 Miller DT, Williams DR, Morris GM, Liang J. Images of cone photoreceptors in the living human eye. Vis Res 1996; 36(8): 1067-1079.

8 Roorda A, Williams DR. The arrangement of the three cone classes in the living human eye. Nature 1999; 397(6719): 520-522.

9 Roorda A, Romero-Borja F, Donnelly WJ, Hebert TJ, Queener H, Campbell MCW. Adaptive optics scanning laser ophthalmoscopy. Optic Express 2002; 10: 405-412.

10 Kaemmerer M, Mrochen M, Mierdel P, Krinke HE, Seiler T. Clinical experience with the Tscherning aberrometer. J Refract Surg 2000; 16: S584-S587.

11 Buscemi P. Clinical applications of the OPD-Scan wavefront aberrometer/corneal topographer. J Refract Surg 2002; 18(3 Suppl): S385-S388.

12 MacRae S, Fujieda M. Slit skiascopic-guided ablation using the Nidek laser. J Refract Surg 2000; 16(5): S576-S580.
13 Wang L, Wang N, Koch DD. Evaluation of refractive error measurements of the WaveScan wavefront system and the Tracey wavefront aberrometer. J Cataract Refract Surg 2003; 29(5): 970-979.

14 Wang L, Misra M, Pallikaris IG, Koch DD. Comparison of a ray-tracing refractometer, autorefractor, and computerized videokeratography in measuring pseudophakic eyes. J Cataract Refract Surg 2002; 28(2): 276-282.

15 Schwiegerling J. Scaling Zernike expansion coefficients to different pupil sizes. J Opt Soc Am A Opt Image Sci Vis 2002; 19(10): 1937-1945.

16 Campbell CE. Matrix method to find a new set of Zernike coefficients from an original set when the aperture radius is changed. J Opt Soc Am A Opt Image Sci Vis 2003; 20(2): 209-217.

17 Goldberg KA, Geary K. Wave-front measurement errors from restricted concentric subdomains. J Opt Soc Am A Opt Image Sci Vis 2001; 18(9): 2146-2152.

18 Oshika T, Miyata K, Tokunaga T, Samejima T, Amano S, Tanaka $\mathrm{S}$ et al. Higher order wavefront aberrations of cornea and magnitude of refractive correction in laser in situ keratomileusis. Ophthalmology 2002; 109(6): 1154-1158.

19 Kuroda T, Fujikado T, Maeda N, Oshika T, Hirohara Y, Mihashi T. Wavefront analysis of higher-order aberrations in patients with cataract. J Cataract Refract Surg 2002; 28(3): 438-444.

20 Oshika T, Mimura T, Tanaka S, Amano S, Fukuyama M, Yoshitomi $\mathrm{F}$ et al. Apparent accommodation and corneal wavefront aberration in pseudophakic eyes. Invest Ophthalmol Vis Sci 2002; 43(9): 2882-2886.

21 Liang CL, Juo SH, Chang CJ. Comparison of higher-order wavefront aberrations with 3 aberrometers. J Cataract Refract Surg 2005; 31(11): 2153-2156.

22 Salmon TO, West RW, Gasser W, Kenmore T. Measurement of refractive errors in young myopes using the COAS Shack-Hartmann aberrometer. Optom Vis Sci 2003; 80(1): 6-14.

23 Gobbe M, Guillon M, Maissa C. Measurement repeatability of corneal aberrations. J Refract Surg 2002; 18(5): S567-S571.

24 Davies N, Diaz-Santana L, Lara-Saucedo D. Repeatability of ocular wavefront measurement. Optom Vis Sci 2003; 80(2): 142-150.

25 Davies LN, Mallen EA, Wolffsohn JS, Gilmartin B. Clinical evaluation of the Shin-Nippon NVision-K 5001/Grand Seiko WR-5100K autorefractor. Optom Vis Sci 2003; 80(4): 320-324.

26 Allen PM, Radhakrishnan H, O'Leary DJ. Repeatability and validity of the PowerRefractor and the Nidek AR600-A in an adult population with healthy eyes. Optom Vis Sci 2003; 80(3): 245-251. 